\title{
Influence du froid sur les tsé-tsé et ses indications
}

\author{
par L. MAILLOT
}

\begin{abstract}
RESUME
Des expériences de refroidissement de tsé-tsé d’élevage ( $G$. morsitans, $G$. austeni et $G$. tachinoides) ont été réalisées en chambre froide ou en récipients réfrigérés.

Les différents résultats ont montré que si la température ne s’abaissait pas au-dessous de $5^{\circ} \mathrm{C}\left(41^{\circ} \mathrm{F}\right)$ un refroidissement momentané n'avait qu'une action très faible ou nulłe sur la survie et le pouvoir reproducteur des mouches ainsi traitées.

On peut donc préconiser pour lexpédition par avion vers un centre d'élevage ou d'études le transport des mouches tsé-tsé en récipients isolants, avec des produits frigorigènes réalisant ainsi les conditions optimales de température observées dans les expériences décrites.
\end{abstract}

Les élevages autonomes de mouches tsé-tsé surtout ceux pratiqués en Europe nécessitent un apport initial important d'adultes ou de pupes dont l'expédition doit être faite dans les meilleures conditions possibles. Le transport dans des récipients réfrigérés paraît constituer la solution idéale. Les expériences réalisées ont montré que la limite inférieure de température ne devait pas s'abaisser au dessous de $5^{\circ} \mathrm{C}$ $\left(41^{\circ} \mathrm{F}\right)$ et que des produits frigorigènes actuellement en vente courante dans le commerce pouvaient être utilisés avec profit.

Dans un précédent article (6) l'auteur avait exposé les divers procédés d'expédition rapide de mouche tsé-tsé maintenues à basse température.

Une série d'expériences (de juillet 1966 à septembre 1969) ont été réalisées au laboratoire d'Entomologie avec des mouches d'élevage (adultes et pupes) pour étudier les effets du refroidissement sur celles-ci.

Les températures des différentes expériences s'échelonnent de 18 à $2^{\circ} \mathrm{C}\left(64\right.$ à $\left.36^{\circ} \mathrm{F}\right)$, la température de la salle d'élevage étant voisine de $25^{\circ}\left(77^{\circ} \mathrm{F}\right)$.
Les divers procédés de refroidissement ont consisté à garder les tsé-tsé ou dans une chambre froide ou dans des récipients isolants avec des produits congelés, en particulier certains produits frigorigènes maintenant en vente courante dans le commerce.

Les mouches ou pupes ainsi refroidies sont ensuite gardées dans la salle d'élevage à $25^{\circ} \mathrm{C}$ et 70 à 80 p. 100 d'humidité relative.

Les diverses expérimentations se sont proposées d'étudier :

1. la survie de mouches adultes gorgées avant refroidissement et par la suite ou laissées à jeun jusqu'à leur mort ou réalimentées régulièrement après l'épreuve;

2. la survie de femelles fécondées exposées au froid et leur taux de ponte;

3. le taux d'éclosion des pupes refroidies.

Dans chacune de ces expériences un lot témoin était gardé à $25^{\circ}$ ou dans la salle d'élevage ou dans un récipient isolant.

Enfin on a étudié la valeur isolante de différents récipients (boîtes en mousse de polystyrène, bouteilles isolantes de type " thermos", 
emballages divers) par refroidissement ou réchauffement.

\section{Survie des mouches exposées au froid et réalimentées après l'expérience}

a) Juillet 1966: 2 lots de 10 G. morsitans mâles âgés de 12 à 17 jours récemment gorgés sur cobaye sont placés chacun dans un récipient identique en m.d.p. ( $\left.{ }^{*}\right)$ pendant 48 heures. Dans l'un la température est de $25^{\circ}$, dans l'autre de $18^{\circ}$ environ. On observe que la survie moyenne du lot refroidi est notablement augmentée : la survie moyenne du lot refroidi est de 54 jours, du lot témoin de 33 jours; la survie maximale du lot refroidi est de 67 jours, du lot témoin de 63 jours. (La température de $18^{\circ} \mathrm{C}$ est la limite au dessous de laquelle $G$. morsitans perd toute activité. GLASGOW 1963 (5).

b) Juin $1969: 2$ lots de 9 G. austeni mâles âgés de 3 jours ont depuis leur éclosion été quotidiennement nourris sur lapin; un des lots est placé dans un récipient isolant en m.d.p. pendant 2 h 20 avec un produit frigorigène de marque Handy Ice, non entièrement congelé; la température minimale atteint $10^{\circ}$. Trois mois après l'épreuve, la mortalité dans le lot refroidi atteint 66 p. 100 , elle est nulle dans le lot témoin. Les résultats de cette dernière expérience s'expliquent peut-être par une plus grande fragilité des mâles et leur jeune âge. BURNETT 1957 (2) constate, pour des tsé-tsé soumises à des températures de 1,2 à $9^{\circ} \mathrm{C}$, une mortalité légèrement plus élevée chez les mâles et d'autre part qu'une courte exposition à une température allant de 9 à $10^{\circ}$ semble réduire la survie des mouches âgées de 5 jours tandis qu'elle est sans effet sur des mouches âgées de 16 jours.

c) Juin 1969: 2 lots de 10 G. austeni femelles âgées de 4 jours ont été depuis leur éclosion quotidiennement nourries sur lapin; un des lots est placé dans un récipient isolant comme dans la précédente expérience pendant 2 h 40 avec le produit Handy Ice congelé; la température minimale atteint $8^{\circ}$. Trois mois après, la mortalité dans le lot témoin est de 30 p. 100 , de 10 p. 100 dans le lot refroidi. La différence des deux taux de mortalité n'est pas significative, la courte exposition à $8^{*}$ est

$\left({ }^{*}\right)$ Mousse de polystyrène. dans ce cas sans effet nuisible appréciable sur les mouches traitées.

\section{Survie de mouches exposées au froid et Iaissées à jeun après l'épreuve}

a) Octobre 1967:40 G. tachinoides et 40 G. morsitans (vieux mâles d'âge indéterminé) récemment nourries sur cobaye sont placées par lots de 10 à 20 dans une chambre froide pendant des périodes de 1 heure, 2 heures et 24 heures, la température variant de 2 à $6^{\circ}$. Leur mortalité après l'épreuve est comparée à celle de deux lots témoins de 20 mouches des deux espèces, de même sexe, sensiblement du même âge et nourries dans les mêmes conditions avant l'épreuve. La mortalité :

- est plus forte dans les lots refroidis;

- plus élevée chez $G$. tachinoides;

- chez G. tachinoides, dans le lot refroidi, la survie moyenne est de 1 jour environ, la survie maximale de 4 jours; les chiffres correspondants sont dans le lot témoin respectivement 3 et 6 jours;

- chez G. morsitans, dans le lot refroidi 24 heures, la survie moyenne est de 2 jours environ, la survie maximale de 5 jours, les chiffres correspondants sont respectivement dans le lot refroidi 1 heure : 3 et 5 jours, dans le lot témoin 4 et 7 jours.

b) Juin 1969: 2 lots de 38 G. morsitans mâles âgés d'environ 13 jours sont gorgés sur oreilles du lapin; un des lots est placé dans un récipient en m.d.p. pendant 6 heures avec le produit Handy Ice congelé; la température minimale atteint $8^{\circ}$. La survie moyenne et la survie maximale sont identiques dans le lot témoin et le lot refroidi. La survie moyenne du lot témoin est de 5,73 jours, du lot refroidi de 5,79 jours, la survie maximale dans les deux lots est de 10 jours.

c) Juillet 1969: 2 lots de 25 G. morsitans mâles de 13 jours environ sont nourris sur oreilles de lapin; un des lots est placé dans un récipient de m.d.p. avec le produit Frescal congelé pendant 6 heures; la température atteint le minimum de $4^{\circ}$. La survie moyenne et la survie maximale sont identiques dans les deux lots. La survie moyenne du lot témoin est de 8,44 jours, du lot refroidi de 9,32 jours, la survie maximale est de 12 jours dans les deux lots. 
Les chiffres des deux dernières expériences $\mathrm{y}$ compris ceux des lots témoins sont légèrement inférieurs à ceux obtenus par JACK 1939 cité par BUXTON pour les $G$. morsitans maintenues à $18^{\circ}$ et 80 p. 100 d'H.R. (3).

\section{Survie de femelles fécondées et taux de ponte après refroidissement}

a) Novembre 1967: 2 lots de 45 G. morsitans femelles fécondées, sensiblement du même âge, sont nourries sur cobaye; l'un d'eux est subdivisé en 3 lots qui sont laissés à la chambre froide respectivement 5,10 et 30 minutes, la température variant de 2 à $6^{\circ}$.

Comparativement au lot témoin, survie et taux de ponte sont plus faibles dans les lots refroidis et d'autant plus faibles que le temps d'exposition au froid a été plus long. Un mois après l'expérience le taux de mortalité atteint chez les mouches témoins 18 p. 100 , chez les mouches refroidies 5 minutes 42 p. $100,10 \mathrm{mi}-$ nutes 31 p. $100,1 / 2$ heure 41 p. 100 . Les mouches témoins ont pondu 54 pupes, les mouches réfrigérées 28 , les mouches témoins ont pondu en moyenne par période de $60 \mathrm{j}$. et par femelle 2,7 pupes, les mouches refroidies 1,6 pupes.

b) Janvier 1968: 2 lots sont constitués par 24 G. morsitans femelles fécondées âgées. L'un des lots est mis dans une cage Roubaud, celleci avec un thermomètre à maxima et minima est placée dans un emballage (carton, papier, coton, compresses) que l'on garde en chambre froide pendant 17 heures, la température dans l'emballage descend jusqu'à $5^{\circ}$.

Dix-huit jours après :

- la mortalité est sensiblement identique dans les deux lots;

- le taux de ponte est un peu plus élevé dans le lot témoin;

- ultérieurement, le taux d'éclosion des pupes est sensiblement le même quelle que soit leur origine (pupes de mouches témoins ou pupes de mouches refroidies).

Les chiffres sont les suivants: la mortalité est de 17 p. 100 dans le lot témoin, de 21 p. 100 dans le lot refroidi; les femelles du lot témoin ont pondu 28 pupes, celles du lot refroidi 26 pupes. Le taux d'éclosion des premières pupes (pondues par les femelles témoins) est de 89 p. 100 , du 2 " lot de pupes (pondues par les femelles refroidies) de 92 p. 100.

Le refroidissement à $5^{\circ}$ ne paraît pas avoir entraîné de modifications de la survie ni de la reproduction.

\section{Action du froid sur les pupes}

a) Novembre 1967 : un lot de 41 pupes de G. morsitans d'âge connu sont placées à la chambre froide dans les mêmes conditions que les mouches de l'expérience 3 à 5 minutes, 10 minutes et 30 minutes entre $2^{\circ}$ et $6^{\prime \prime} \mathrm{C}$. Le lot témoin comprend 56 pupes de G. morsitans d'âge connu.

Le taux d'éclosion est sensiblement le même dans les deux lots. Le cycle nymphal est identique chez les pupes femelles dans les deux lots, un peu allongé dans le lot des pupes mâles refroidies.

b) Janvier 1968: Les conditions d'expérience sont les mêmes qu'en 3 b; 2 lots comprennent chacun 15 pupes de $G$. morsitans pondues sensiblement à la même époque, l'un d'eux est maintenu 17 heures à $5^{\circ}$, dans les deux lots, lot refroidi et lot témoin le taux d'éclosion est identique soit $13 / 15$ ou 87 p. 100 .

\section{CONCLUSIONS}

1. Un abaissement temporaire de la température qui ne descend pas au-dessous de 4 à $5^{\circ}$ ne modifie pas et dans certains cas paraît même allonger la survie des mouches refroidies (sauf dans l'expérience $1 \mathrm{~b}$ ). Un tel abaissement momentané de température n'a pas d'action sur le pouvoir reproducteur des femelles fécondées : taux de ponte et taux d'éclosion des pupes pondues; il en est de même pour les pupes dont le taux d'éclosion n'est pas modifié.

2. Au-dessous d'une température de 4 à $5^{\circ}$ la survie des mouches refroidies est abrégée; le taux de ponte paraît diminué chez les femelles fécondées mais l'action de ces températures basses paraît nulle ou moindre pour les pupes.

3. Le fait de nourrir les mouches avant refroidissement paraît nécessaire pour obtenir de bons résultats. 
4. Les résultats observés avec divers proJuits réfrigérants sont identiques à ceux obtenus en chambre froide.

5. Un petit nombre d'essais ont permis de constater pour des intervalles de temps, il est vrai assez longs, que les récipients isolants utilisables n'avaient (à vide : en l'absence de tout produit réfrigérant) qu'une très faible capacité d'isolement thermique vis-à-vis du refroidissement et surtout du réchauffement.

6. Des deux produits réfrigérants utilisés, le produit danois Handy Ice convenablement congelé fournit au début une température de 8 à $10^{\circ}$, avec une action de plus longue durée (très vraisemblablement du fait du plus grand volume de produit utilisé) que le produit. Frescal (fabriqué en France) qui donne une température minimale de 4 à $6^{\circ}$. Ce dernier produit semble s'être montré dans les expériences réalisées d'une plus grande efficacité pour la survie des mouches, efficacité peut-être attribuable au degré de température atteint.

7. Un produit vendu en bidons par la maison Style a présenté, après essais pratiqués au laboratoire d'Entomologie, un point de congélation de $5^{\circ}$, le fabricant indique une durée d'action de 12 heures.

\section{INDICATIONS}

L'emploi de produits réfrigérants, tels que ceux expérimentés est à conseiller pour le transport des tsé-tsé soit par voie aérienne ou d'Afrique en Europe ou en Afrique d'une région à l'autre, soit éventuellement pour des trajets terrestres plus ou moins longs sur lesquels la mortalité peut être en certains cas très élevée (PEEL et CHARDOME, 1958 (7).

Le produit frigorigène sera mis dans le tiroir à glace d'un réfrigérateur jusqu'à congélation totale du produit, ensuite son contenant est rapidement lavé et essuyé pour éliminer tout dépôt de givre, le produit est alors placé dans le récipient isolant destiné au transport des mouches.

Il existe des frigorigènes contenus dans des sachets en tissu caoutchouté, leur emploi est à déconseiller, étant donné l'action nocive connue du caoutchouc sur les tsé-tsé, les sachets de plastique paraissent quelquefois fragiles, le conditionnement en bidons est peut-être en certains cas préférable.

La bouteille de type «thermos" habituel semble avoir le pouvoir isolant le plus marqué, mais elle est souvent contre indiquée par sa fragilité surtout pour certains voyages en auto par des routes cahoteuses.

Les récipients en mousse de polystyrène (sous réserve de ne pas être exposés à des variations de température fortes et prolongées) constituent la meilleure solution actuelle.

Des récipients en mousse de polyuréthane existent maintenant dans le commerce mais l'auteur n'a pu expérimenter leur pouvoir d'isolement thermique.

Enfin des emballages improvisés pourront rendre service sur de courtes distances de transport (BRENNAN et MAIL, 1954 (1).

\section{SUMMARY}

Effects of low temperatures upon tsetse flies and practical application

A number of experiments in cooling laboratory-bred tsetse flies ( $G$. morsitans, $G$. austeni and $G$. tachinoides) have been carried out in cold rooms or refrigerated containers.

The results obtained have shown that if the temperature does not fall below $5^{\circ} \mathrm{C}\left(41^{\circ} \mathrm{F}\right)$, momentary cooling has but little or no effect on the longevity and breeding potential of the flies subjected to such conditions.

It is possible accordingly, for the purpose of dispatching tsetse flies by air to a breeding or research center, to recommend that they be transported within insulated containers using cooling media and thus complying with the optimum temperature conditions recorded in the experiments described. 


\section{RESUMEN}

\section{Influencia del frio sobre las tsetse $y$ sus indicaciones}

Se realizaron experiencias de enfriamiento de tsetse de cria $(G$. morsitans, $G$. austeni y $G$. tachmoides) en cámara fría o en recipientes refrigerados. Los diferentes resultados mostraron que si la temperatura no bajaba más bajo de $5^{\circ} \mathrm{C}\left(41^{\circ} \mathrm{F}\right)$, un enfriamento momentáneo tenia solo una acción poco importante o ninguna sobre la supervivencia y el poder de reproducción de las moscas asi tratadas.

Se puede preconizar, para la expedición por avión hacia un centro de cria o de estudios, el transporte de las moscas tsetse en recipientes aisladores, con productos frigorigenos, realizando así las condiciones optimas de temperatura observadas en las experiencias descritas.

\section{BIBLIOGRAPHIE}

1. BRENNAN (J M.), MAIL (C.A.), «Une technique pour l'expédition des moustiques vivants en particulier de culex tarsalis » (A technic for shipping live mosquitoes with particular reference to culex tarsalis), Science, New York, 1954, 119 (3092) 443-44.

2. BURNETT (G E.) « La relation entre l'âge et la résistance au froid chez les tsé-tsé et la valeur de la congélation pour le transport de tsé-tsé destinées à l'expérimentation" (The relation between age and cold resistance in tsetse flies and the value of chilling when transporting tsetse for experiments), Proc. R. ent. Soc. Lond. (A.), 1957, 32 (4-6) 53-58.

3. BUXTON (P.A.), "L'histoire naturelle des tsé-tsé. Un exposé de la biologie du genre Glossina
(Diptera)». (The natural history of tsetse flies. An account of the biology of the genus Glossina (Diptera), London, H.K. Lewis, 1955.

4. GEIGY (R.), "Elevage de Glossina palpalis». Acta trop. 1948, 5, 201-18.

5. GLASGOW (J.P.), " La distribution et l'abondance des tsé-tsé » (The distribution and abundance of tsetse), Oxford, Pergamon Press. 1963.

6. MAILLOT (L.), « Expédition de glossines adultes à basse température ", Rev. Elev. Méd vét. Pays trop, 1963, 16 (3) 371-372.

7. PEEL (E) et CHARDOME (M.), «Observations sur les élevages de Glossina morsitans West. au laboratoire », Ann. Soc. belge Med. trop., 1958, 38, 5, 961-964. 